\title{
Comments to the article by Wu: Retrograde dynamic locked nailing for valgus knee correction: a revised technique
}

\author{
Sukesh Rao Sankineani • Ramakant Kumar • \\ Ram Prasad Kancherla
}

Received: 10 February 2012 / Accepted: 14 February 2012 / Published online: 3 March 2012

(C) Springer-Verlag 2012

We read with great interest the work of Chi-chuan $\mathrm{Wu}$ on the use of retrograde dynamic locked nailing for valgus deformity of knee [1]. We would like to congratulate the author for devising a new technique for this deformity. The author has concluded that the use of intramedullary nail provides better biomechanics when compared to the use of plate and that patients have better symptomatic relief. However, we would like to bring forth a few points to the authors notice:

1. Most of the patients were young (average age of 28 years) in the study group when compared to the studies in which plating was used in whom the predominant reason for the osteotomy was osteoarthritis. So, for the outcome of time to union, postoperative satisfaction would be biased. It would have been better if the author would have created a control group in whom plating is used to overcome the bias.

2. The author did not comment on the stability of the implant in this study. Ito et al. [2] concluded that retrograde nail was noted to provide equal or greater stability than the plate, except when large torsional loads are anticipated. As the patient population in the study group was comprised of young individuals with high impact activities, concern regarding the stability of the implant exists.

3. The use of retrograde nails further entails potential risk of knee sepsis, damage to articular cartilage, and ligaments while inserting the nail.

We conclude that the use of retrograde nail to correct the valgus deformity in young adults is a promising technique. However, a comparative trial with a longer follow-up would further shed more light on the advantages of this technique over plating.

\section{References}

1. Wu CC (2012) Retrograde dynamic locked nailing for valgus knee correction: a revised technique. Int Orthop. doi:10.1007/s00264012-1495-8

2. Ito K, Grass R, Zwipp H (1998) Internal fixation of supracondylar femoral fractures: comparative biomechanical performance of the 958 plate and two retrograde nails. J Orthop Trauma 4:259-266

S. R. Sankineani $(\bowtie) \cdot$ R. Kumar $\cdot$ R. P. Kancherla

Department of Orthopaedics,

All India Institute Of Medical Sciences,

New Delhi, India

e-mail: sukeshrao.sankineni@gmail.com 